\title{
Plasma miR-124 Is a Promising Candidate Biomarker for Human Intracerebral Hemorrhage Stroke
}

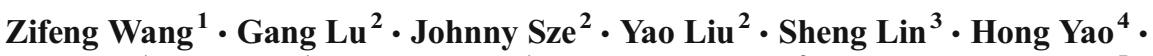 \\ Ji Zhang ${ }^{1}$ - Dan Xie ${ }^{1}$ - Quentin Liu ${ }^{1}$ - Hsiang-fu Kung ${ }^{2}$ - Marie Chia-mi Lin ${ }^{5}$. \\ Wai Sang Poon ${ }^{2}$
}

Received: 2 January 2017 / Accepted: 12 October 2017 / Published online: 3 November 2017

(C) The Author(s) 2017. This article is an open access publication

\begin{abstract}
Stroke causes death or long-term disabilities and threatens the general health of the population worldwide. Recent studies have suggested that miRNAs are dysregulated and can be used as biomarkers for diagnosis and prognosis in stroke. The intracerebral hemorrhage (ICH) accounts for 15\% of all the stroke cases. However, at present, little is known regarding the functions and clinical implications of miRNAs in ICH. In the present study, we established the collagenase-induced rat ICH model to mimic human ICH syndrome. We profiled the expression of 728 rat miRNAs at different time points in rat brain tissues and plasma post-ICH and identified a set human brain-enriched miRNAs that had changed expression level in the plasma of rat ICH. Among them, the expression levels of miR-124 displayed significantly synchronous alterations in rat plasma and brain tissue during ICH progression. They were significantly elevated at the acute injury phase (day 1 and 2), gradually decreased during
\end{abstract}

Zifeng Wang and Gang Lu contributed equally to this work.

Marie Chia-mi Lin

momlin@163.com

Wai Sang Poon

wpoon@surgery.cuhk.edu.hk

1 State Key Laboratory of Oncology in South China, Collaborative Innovation Center of Cancer Medicine, Sun Yat-sen University Cancer Center, Guangzhou, China

2 Brain Tumor Centre and Division of Neurosurgery, Department of Surgery, Faculty of Medicine, The Chinese University of Hong Kong, Prince of Wales Hospital, Shatin, Hong Kong, China

3 Laboratory of Medical Genetics, Shenzhen Research Institute of Population and Family Planning, Shenzhen, China

4 Jiangsu Eng. Laboratory of Cancer Biotherapy, Xuzhou Medical College, Xuzhou, China

5 Academy of Medical Science, Zhengzhou University, Zhengzhou, Henan, China the delayed recovery phase (day 7, 14 and 30), and finally restored to normal levels at late recovery phase (day 60 ). We further determined the plasma expression profile of miR-124 from human ICH patients. Similar to the pattern observed in rat ICH model, our results indicated that immediately after patients reached the hospital, the average plasma concentrations of miR-124 increased more than 100-fold in $24 \mathrm{~h}$, then decreased gradually on day $2,7,14$ and to near normal level on day 30 . Taken together, these results strongly suggested that plasma concentration of miR-124 is a promising candidate biomarker for the early detection and predictive prognosis of human ICH.

Keywords Stroke $\cdot$ Intracerebral hemorrhage (ICH) · Biomarker $\cdot$ Plasma miRNAs $\cdot$ miR-124

\section{Introduction}

Stroke is a cerebrovascular disease and the second leading cause of death, accounting for $9.6 \%$ of the total mortality worldwide [1]. About $80 \%$ of stroke cases are due to cerebral ischemia (ischemic stroke), and $15 \%$ of stroke cases are caused by intracerebral hemorrhage (hemorrhagic stroke, ICH) [2,3]. ICH is resulted from ruptured blood vessel(s) in the brain leading to the pooling of blood in the brain parenchyma and the formation of hematoma [4]. These processes are associated with multiple biological processes including oxidative damage, inflammation, edema formation, neuronal cell death, and dysregulation of multiple genes [5-7]. The mortality of ICH is often as high as 50\% within the first month after $\mathrm{ICH}$, with half of the deaths occurring within $48 \mathrm{~h}$ [8]. Timely diagnosis and treatment are important for ICH patients.

MicroRNAs (miRNAs) are small non-coding RNA molecules (19-30 nt) that are endogenous regulators of gene expression [9]. Many miRNAs are expressed in a tissue- and/or 
cell-specific manner and their expression patterns are reflective of underlying patho-physiologic processes. miRNAs can be detected in serum and plasma in a remarkably stable form, making them desired biomarkers for human diseases [10]. Circulating miRNAs, including miR-1, miR-122, miR-124, miR-133a, miR-192, and miR-208, have been proved to be sensitive and specific markers for monitoring acute tissue injuries [11]. It has been shown that miRNAs are temporally regulated during progression/reperfusion of cerebral ischemia, miR-138 and miR-145 in total blood could be used as diagnostic markers [12]. Similarly, in traumatic brain injury (murine model), correlations have been found between the temporal miRNA expression profile and several biological processes underlying the brain injury [13]. In human patients, Tan et al. has demonstrated that blood miRNA from young ischemic stroke patients (18 to 49 years) could be used to identify the disease progression and the stroke subtype [14]. Recently, miRNA-210 has been shown to be a novel blood biomarker in acute cerebral ischemia [15].

As compared to ischemic stroke, little study related to the role of miRNAs in ICH has been reported. Recently, Liu et al. examined the brain and blood miRNA expression profile changes using a blood infusion (lysed blood, fresh blood, or thrombin) ICH rat model at $24 \mathrm{~h}$ after injury. They found that several miRNAs changed more than 2-fold in both brain and blood, suggesting the possibility of blood miRNAs as ICH biomarkers [16]. To date, the function and clinical implications of miRNA in human ICH patient are not well understood. In the present study, we established the collagenaseinduced rat ICH model to mimic human ICH syndrome and then measured 728 different miRNAs expressed in plasma samples and collagenase-damaged brain tissues at different time points. We further identified the brain-specific miR-124 as a promising candidate biomarker in ICH patient plasma for early detection and of intracerebral hemorrhage.

\section{Materials and Methods}

\section{The Rat Collagenase-Induced ICH Model}

All procedures involving animals were performed to minimize pain or discomfort in accordance with current protocols approved by the Animal Research Ethics Committee of The Chinese University of Hong Kong. A cohort of 21 male Sprague-Dawley rats with a mean age of 12 weeks and body weight of $220 \mathrm{~g}$ were used.

Subject rats were randomly separated into two groups, one injected with PBS (sham surgery control, $n=3$ ) and the other injected with collagenase (ICH model, $n=18$ ). These rats were first anesthetized by intraperitoneal injection of $30 \mathrm{mg} /$ kg ketamine (Alfasan, FarmaVet SA, Bucharest, Romania) and $4 \mathrm{mg} / \mathrm{kg}$ xylazine (Alfasan) then fixed to a stereotactic frame (David Kopf Instruments, Tujunga, CA). A surgical incision was made in the midline of the skull. Using a microdrill, a puncture was made at $0.2 \mathrm{~mm}$ posterior to the bregma and $3 \mathrm{~mm}$ left lateral to the midline. A Hamilton syringe with a 26-gauge needle was inserted $6 \mathrm{~mm}$ below the skull surface, and $1.5 \mu \mathrm{L}$ of PBS or 0.21 collagenase digestive units (Sigma) was slowly injected into the internal capsule over $5 \mathrm{~min}$. The needle was allowed to remain in place for a further $5 \mathrm{~min}$ before gentle withdrawal. The burr hole was sealed with bone wax, and the skin incision was sutured. Rats were allowed access to food and water ad libitum in isolator cages at $25{ }^{\circ} \mathrm{C}$ under a 12 -h light/dark cycle. This was basically a collagenase-induced ICH into the striatum based on Rosenberg et al. [17] and MacLellan et al. [18]. The sham surgery group was anesthetized and sacrificed at 1 day post-PBS injection, while the ICH model groups were operated at days $1,2,7,14,30$, and 60 post-collagenase injection ( $n=3$ at each time point). The brain hematoma and the cardiac blood were harvested, and the blood was further separated into blood cells and plasma fractions.

\section{Experimental Design}

The experimental design of this study is summarized in Fig. 1. For the rat ICH studies (Fig. 1 left panel), we first established the rat model of collagenase-induced ICH, which is believed to mimic human ICH syndrome $[17,18]$. Then, the plasma and brain tissue samples were collected, with the sham surgery group at day $1(n=3)$ and the ICH model groups $(n=18)$ at days $1,2,7,14,30$, and 60 ( $n=3$ per time point) postcollagenase injection. For human ICH studies, as shown in Fig. 1 right panel, 20 patients who were diagnosed as $\mathrm{ICH}$ and hospitalized in the Prince of Wales Hospital in The Chinese University of Hong were recruited in this study. The first blood samples were collected within $24 \mathrm{~h}$ after the admission, and then on day 2, 7, and 30. EDTA blood was centrifuged at $1600 \mathrm{~g}$ for $10 \mathrm{~min}$ at $4{ }^{\circ} \mathrm{C}$, and plasma was transferred to new tubes followed by further centrifugation at $16000 \mathrm{~g}$ for 10 min at $4{ }^{\circ} \mathrm{C}$. Then, the supernatants were collected into fresh tubes and stored at $-20^{\circ} \mathrm{C}$ until ready for experiments.

\section{Plasma miRNA Extraction from Human ICH Patients and Rat ICH Models}

The human and rat plasma miRNAs were extracted according to the procedure of EKO Ng et al. [19]. Briefly, total RNA containing small RNA was extracted from $500 \mu \mathrm{L}$ of plasma using Trizol LS reagent (Invitrogen, CA, USA) and miRNeasy Mini Kit (Qiagen, Hilden, Germany) according to the manufacturer's protocol with the following modifications: Trizol LS reagent was added to plasma samples in volumetric ratio about 4:3. After phase separation by chloroform addition and centrifugation, 1.5 volumes of $100 \%$ ethanol was 


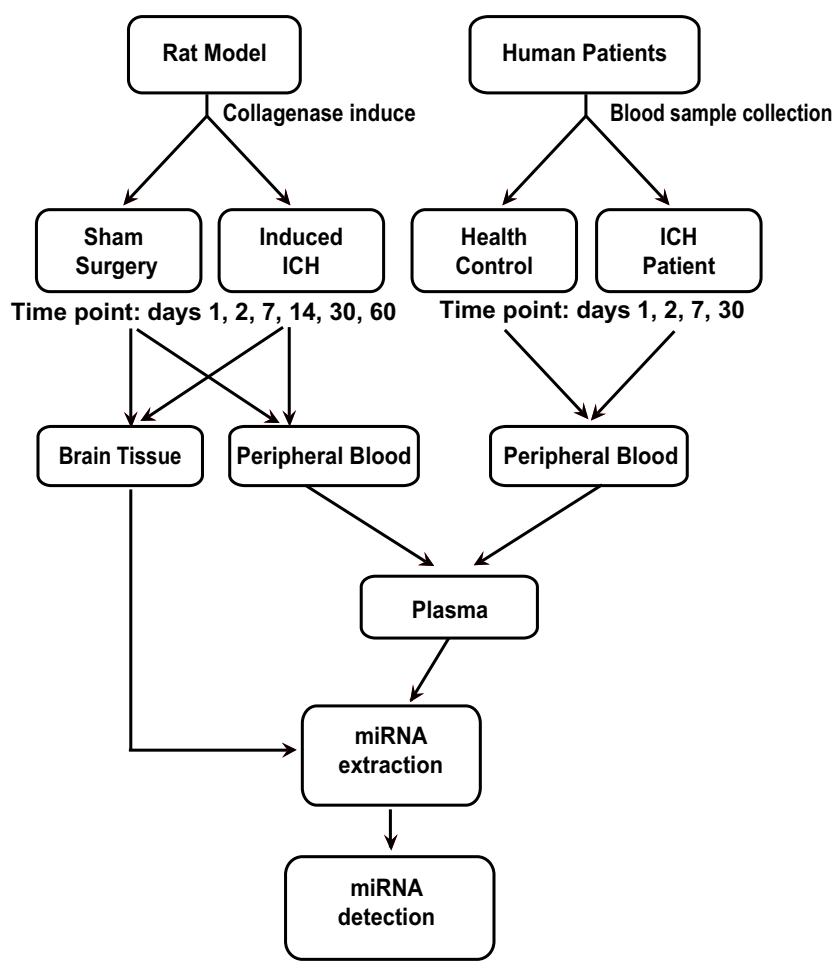

Fig. 1 Flow diagrams showing experimental design of this study. Collagenase-induced preclinical rat model of ICH was first established and evaluated. Brain tissue and plasma miRNAs were extracted and measured and then confirmed in human patient plasma

added to the aqueous phase, and the mixture was loaded into miRNeasy column according to the manufacturer's instructions. A supplement of the bacteriophage MS2 (Roche) was added to increase the yield of total RNA extracted.

\section{miRNA Extraction from Rat Brain Tissues}

For miRNA extraction from brain tissues (hippocampus), the total RNA was extracted using Trizol (Invitrogen) in accordance with the manufacturer's instructions. The final elution volume was $30 \mu \mathrm{L}$ and the concentrations of all RNA samples was quantified by NanoDrop 1000 (NanoDrop, Wilmington, Delaware, USA). RNA samples were stored at $-80^{\circ} \mathrm{C}$ before use.

\section{miRNA Profiling by Locked Nucleic Acid (LNA) qPCR Assay}

To achieve high sensitivity and specificity profiling of miRNAs in less abundant medium, cDNA synthesis and quantitative real-time PCR (qPCR) were performed using the miRCURY LNATM Universal RT microRNA PCR system (Exiqon, Denmark) according to the manufacturers' instructions. In brief, miRNA samples from the same group $(n=3)$ were pooled together for miRNA microarray profiling. The RNA was tailed with a poly(A) sequence at their 3'end and then reverse transcribed into cDNA using a universal poly(T) primer with a $3^{\prime}$ end degenerate anchor and a 5 'end universal tag.

The qPCR screen of the miRNAome was performed with microRNA ready-to-use PCR mouse and rat panel I + II V2 R with primers for 752 rodent miRNAs ( 728 of these are present in rat, Exiqon, Denmark). The reactions were performed on an ABI 7900 thermocycler (Applied Biosciences, 10 min at $95^{\circ} \mathrm{C}$ with 45 amplification cycles at $95{ }^{\circ} \mathrm{C} 10 \mathrm{~s}, 60^{\circ} \mathrm{C} 1 \mathrm{~min}$ ) according to the manufacturer's instructions. The qPCR data were analyzed using the $2^{-\Delta \mathrm{Ct}}$ quantitative method, where $\Delta \mathrm{Ct}=\mathrm{Ct}$ target $-\mathrm{Ct}$ global mean. Expression intensities were $\log 2$ transformed and median-centered by subtracting the median value of each array from each intensity value. Hierarchical clustering was performed with complete linkage and Euclidean distance measurements with HCE 3.5 software [20].

Technical confirmation of the candidate miRNAs from the miRNAome screen was carried out in triplicate using the same RNA template and primers as for the screen. The primers are based on Exiqon's patented LNA ${ }^{\mathrm{TM}}$ technology, and all primers were commercially obtained from Exiqon, Denmark. The reactions were performed in a 96-well plate format (Pick-\&-Mix panel from Exiqon) on a Roche Lightcycler 480 according to the manufacturer's instructions.

\section{Prediction of miRNAs Target Genes and Biologic Function}

We predicted miRNA target genes by publicly available software TargetScan 5.2 web tool. Then, the candidate targets were further submitted to DAVID algorithm to analyze the pathways involved.

\section{Statistics Analysis}

The data were analyzed by unpaired two-tailed Student's $t$ test and expressed as mean \pm standard deviation (SD). Differences were considered statistically significant at $p$ value $<0.05$.

\section{Results}

\section{Identification of miRNAs with Altered Expression Levels During Rat ICH Progression}

The miRNA profiling was conducted by locked nucleic acid (LNA) qPCR assay as described in Methods. To achieve high sensitivity and specificity for miRNA profiling, the miRCURY LNA $^{\text {TM }}$ Universal RT microRNA PCR was performed. A total of 742 miRNAs were analyzed by the mouse and rat panel-based real-time PCR and LNA-enhanced miRNA-specific primers. To reduce the endogenous errors of high throughput analysis, we first measured the miRNA profiles of the pooled samples 
$(n=3)$ in each experimental group, and the mean values of each group were used for further analysis.

Of the 748 mature Rattus norvegicus (rno)-miRNAs analyzed by the panel, 301 miRNAs were detected in our profiling analysis. Hierarchical clustering analysis showed that samples from brain tissues and plasma, respectively, clustered together (Fig. 2, left panel). Furthermore, the later time points (day 30 and 60 at the recovery phase) clustered together with sham surgery and away from the early time points. In addition, we identified 33 brainspecific (enriched) miRNAs with significantly changed expression levels in the plasma (Fig. 2, right panel).

\section{Identification of Human Brain-Specific miRNAs, miR-124, miR-490, miR-873, and miR-708 with Changed Expression Level in the Plasma of Rat ICH}

An ideal plasma biomarker of brain injury should be abundantly and preferentially produced in the brain, while not present or at low concentrations in the blood and other body fluids. Upon brain injury, such biomarkers are expected to be released into the systemic circulation or other body fluid, where they can be detected in a blood-based assay or assay of another accessible body fluid [11]. From this perspective, the brain specifically enriched miRNA(s) are the best candidate(s) for ICH. By data mining, we first search the miRNAs which are brain-specific and enriched but also weekly expressed in the blood. Pablo Landgraf et al. have detected the expression of more than 600 miRNAs in 147 human tissues and cell lines by next-generation sequencing [21]. We analyzed the expression levels of these miRNAs in the brain and hematopoietic organs. The top 20 human brain-enriched miRNAs were listed as shown in Table 1.

Then, we examined the expression levels of these $20 \mathrm{hu}-$ man brain-enriched miRNAs in our rat ICH model screening. As shown in Fig. 3a, miR-124, miR-490, miR-873, and miR708 are human brain-specific miRNAs with changed expression in the plasma of rat ICH. Since miRNAs exert their function through suppressing gene translation, we predicted these four altered miRNAs by TargetScan 7.0 web tool, respectively. Then, the predicted targets were submitted into DAVID to analyze the enriched biological processes. Many bioprocess concerns in the brain/nervous system development were identified as the top-ranked (Fig. 3b), suggesting that these miRNAs were brain-derived.

The expression patterns of these four miRNAs in the plasma and brain tissues during ICH progression were shown in Fig. 4a. Among these miRNAs, the expression of miR-124, miR-490, and miR-873 expressed lower than 1\% in hematopoietic organs. Among them, miR-124 is most highly expressed (more than 97\%) in the brain (Fig. 4b). Hence, we chose miR-124 as the candidate miRNA for further study.

\section{Brain-Specific/Enriched miR-124 Was Expressed Synchronously in Plasma and Brain Tissue in the Rat ICH Stroke Model}

To comprehensively evaluate the expression pattern of miR-124 in ICH, we monitored the expression of miR-
Fig. 2 miRNA expression profile in rat brain tissue and plasma after ICH. Hierarchical cluster analysis of miRNAs expression profile in brain tissue and plasma of rats subjected to ICH for day 1, 2, 7, $14,30,60$, or to sham surgery. The later time points luster together with sham surgery and away from the early time points (left panel). The miRNAs which were relatively high expressed in brain tissue and significantly changed expression in plasma were displayed in right panel. Red indicates high expression and blue low expression

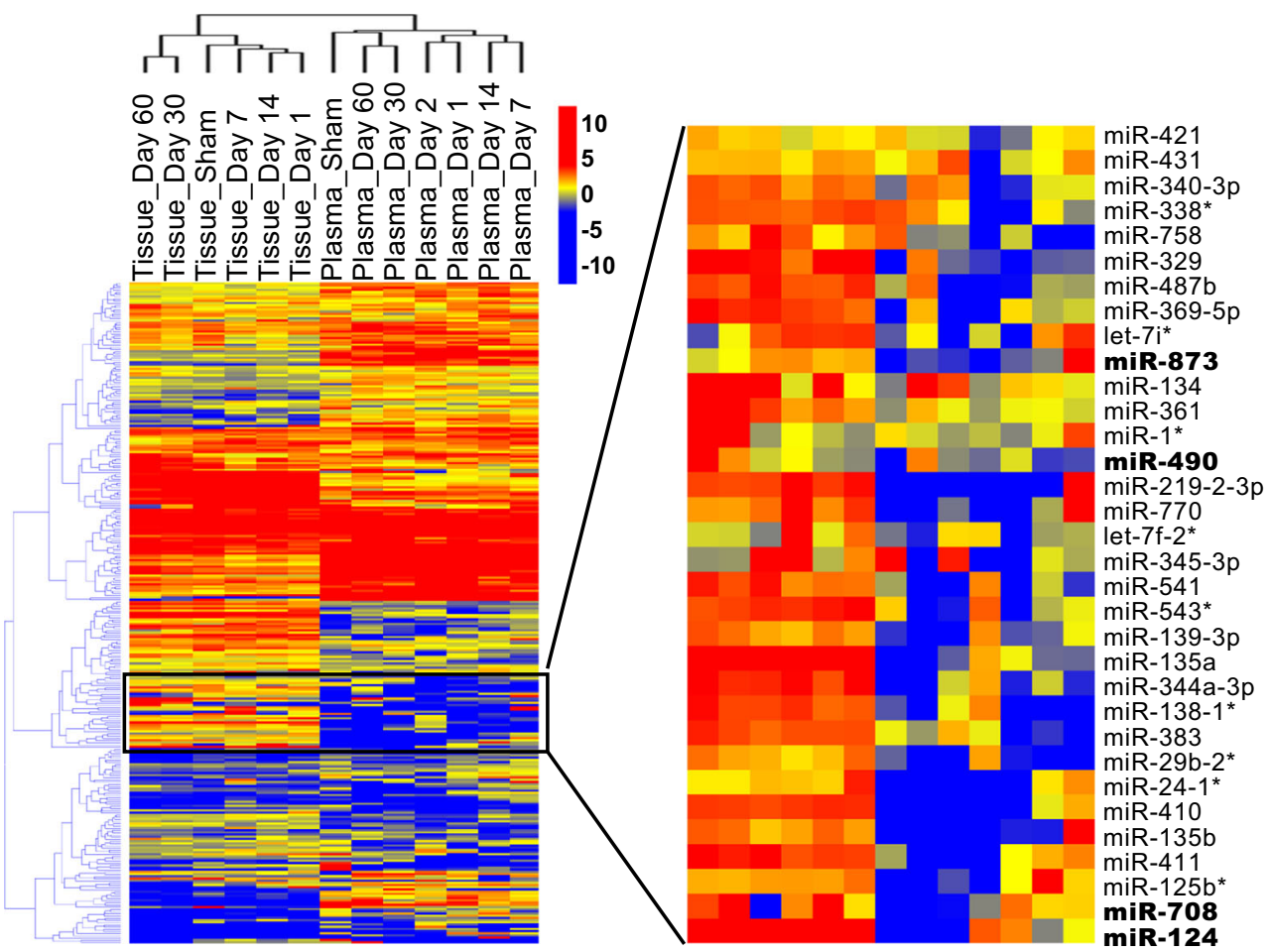


Table 1 Brain-specific miRNAs and their expression in hematopoietic organs

\begin{tabular}{|c|c|c|c|c|c|c|}
\hline \multirow[t]{2}{*}{ miRNA } & \multirow[t]{2}{*}{ Total copies } & \multicolumn{2}{|l|}{ Brain } & \multicolumn{2}{|c|}{ Hematopoietic organs } & \multirow{2}{*}{$\begin{array}{l}\text { Hematopoietic/ } \\
\text { brain ratio }\end{array}$} \\
\hline & & Copies & Percentage $(\%)$ & Copies & Percentage $(\%)$ & \\
\hline hsa-miR-124 & 1613 & 1568 & 97.21 & 14 & 0.87 & 0.0089 \\
\hline hsa-miR-490 & 16 & 14 & 87.50 & 0 & 0.00 & 0.0000 \\
\hline hsa-miR-488 & 53 & 46 & 86.79 & 0 & 0.00 & 0.0000 \\
\hline hsa-miR-873 & 15 & 12 & 80.00 & 0 & 0.00 & 0.0000 \\
\hline hsa-miR-218 & 66 & 40 & 60.61 & 0 & 0.00 & 0.0000 \\
\hline hsa-miR-138 & 79 & 46 & 58.23 & 15 & 18.99 & 0.3261 \\
\hline hsa-miR-153 & 84 & 48 & 57.14 & 24 & 28.57 & 0.5000 \\
\hline hsa-miR-129 & 52 & 29 & 55.77 & 14 & 26.92 & 0.4828 \\
\hline hsa-miR-379 & 103 & 51 & 49.51 & 7 & 6.80 & 0.1373 \\
\hline hsa-miR-136 & 167 & 82 & 49.10 & 7 & 4.19 & 0.0854 \\
\hline hsa-miR-338 & 27 & 13 & 48.15 & 1 & 3.70 & 0.0769 \\
\hline hsa-miR-204 & 23 & 11 & 47.83 & 2 & 8.70 & 0.1818 \\
\hline hsa-miR-708 & 153 & 67 & 43.79 & 20 & 13.07 & 0.2985 \\
\hline hsa-miR-128 & 407 & 173 & 42.51 & 182 & 44.72 & 1.0520 \\
\hline hsa-miR-139 & 27 & 11 & 40.74 & 8 & 29.63 & 0.7273 \\
\hline hsa-miR-99a & 904 & 336.5 & 37.22 & 165 & 18.25 & 0.4903 \\
\hline hsa-miR-92b & 71 & 26 & 36.62 & 6.5 & 9.15 & 0.2500 \\
\hline hsa-miR-877 & 40 & 14 & 35.00 & 6 & 15.00 & 0.4286 \\
\hline hsa-miR-125b & 1317 & 456 & 34.62 & 214 & 16.25 & 0.4693 \\
\hline hsa-miR-137 & 148 & 50 & 33.78 & 0 & 0.00 & 0.0000 \\
\hline
\end{tabular}

The copy number of miRNAs is based on 147 human cells and tissues detected by Pablo Landgraf et al. 2007
124 in both brain tissue and plasma in rat ICH stroke model at six time points, which include acute injury phase (day 1 and day 2), delayed recovery phase (day 7 and day 14), and recovery phase (day 30 and day 60). We first used relative qPCR to detect miR-124 expression in ICH rat brain tissues and plasma. Rno-miR-21 and rno-miR-23b were used as the endogenous control as suggested by the "Norm-finder" algorithm, a modelbased variance estimation predicting the stability of a given miRNA across samples. The qPCR data for the technical confirmation was analyzed using the $2-\Delta \Delta \mathrm{Ct}$ quantitative method.

As shown in Fig. 5a, the expression levels of miR-124 in both brain tissue and plasma were synchronous and significantly elevated at the acute injury phase (day 1 and day 2), gradually decreased during the delayed recovery phase (day 7 and day 14) and recovery phase (day 30). Notably, on day 14 and 30 , the expression levels of miR-124 in both brain tissue and plasma decreased to levels significantly below the normal level (sham group). At later recovery phase (day 60), the expression levels of miR-124 in the brain and plasma returned to normal level.

To further confirm the result, we determined plasma miR124 level using absolute qPCR. As shown in Fig. 5b, using absolute qPCR, similar expression patterns were observed.

\section{The Plasma of Human ICH Patient Samples Exhibited Similar Expression Patterns as Compared to Rat ICH}

To our knowledge, miRNA expression pattern in human ICH patient plasma samples has not been reported. Here, we measured the expression pattern of miR-124 in plasma samples from 20 human ICH patients admitted in the Prince of Wales Hospital (Table 2). As shown in Fig. 6, the relative miR-124 expression level was highest on day 1 ( $n=20,31,765 \pm 14,049$ copies per ul plasma) within $24 \mathrm{~h}$ after patient admission to the hospital, and gradually decreased starting from day $2(n=20,17,176 \pm 9739$ copies per ul plasma) and day 7 ( $n=16,684 \pm 308$ copies per ul plasma), day 30 ( $n=6,206 \pm 45$ copies per ul plasma), as compared to normal healthy individuals $(n=6,163 \pm 23)$. These findings are consistent with the results obtained from the rat ICH preclinical animal study. These results suggested that plasma concentration of miR-124 is a promising candidate biomarker for possible early detection, diagnosis, and prognosis of human $\mathrm{ICH}$.

\section{Discussion}

Currently, two prevalent animal models were used to mimic human ICH syndrome, the collagenase-induced [17] and blood infusion models [22]. However, there are significant 
Fig. 3 miR-124, miR-490, miR873 , and miR-708 are human brain-specific miRNAs and change expression in ICH rat plasma. a Venn diagram with ICH rat model-identified miRNAs and human brain-specific miRNAs. b Target genes of miR-124, miR490, miR-873, and miR-708 were predicted by TargetScan,

respectively. Then, the predicted target genes were submitted into DAVID to analyze the biological process (BP) using Gene Ontology. " $n$ " indicates the gene number in each GO term
A $\mathrm{ICH}$ rat model identified miRNAs

Human brain specific miRNAs
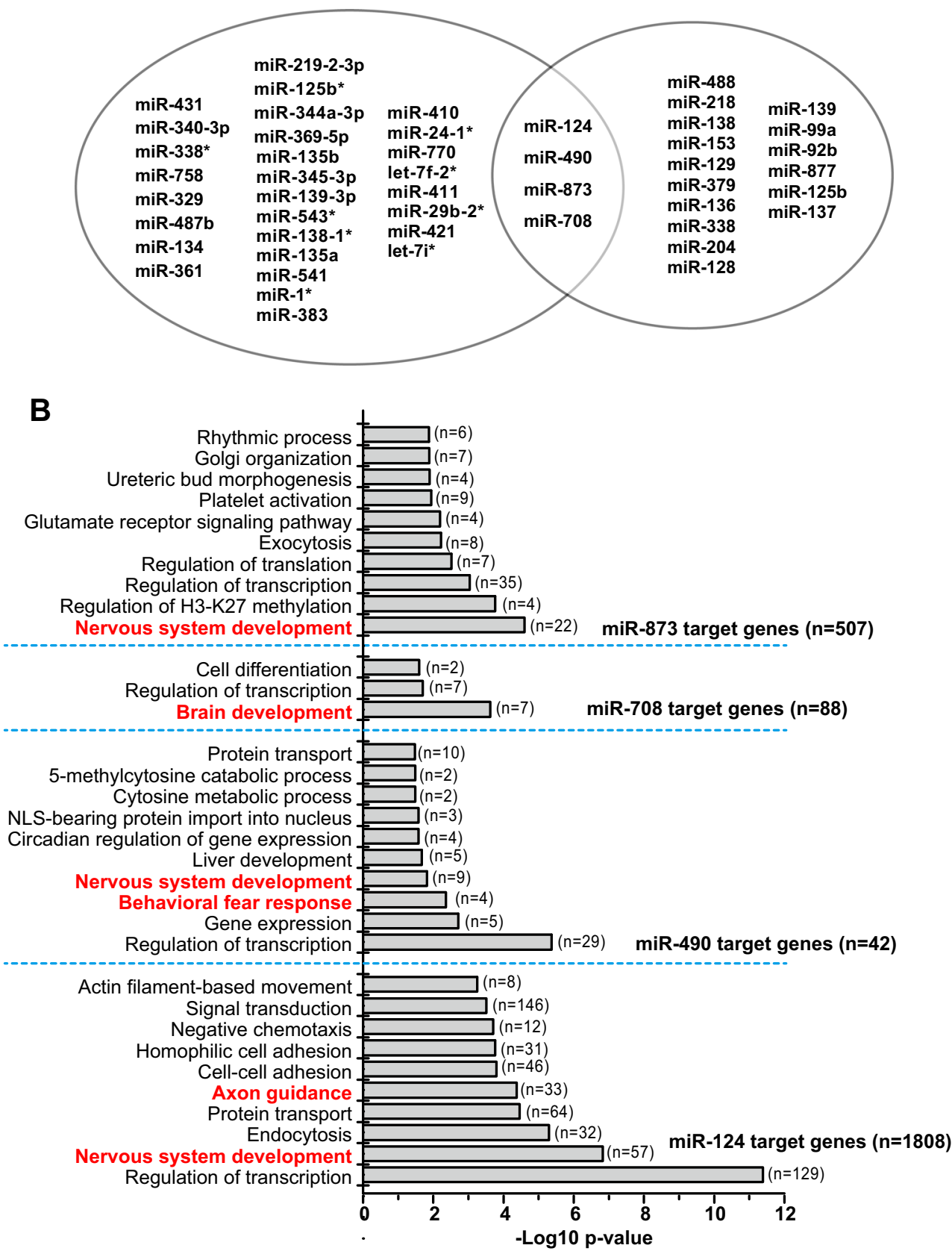

differences between these two models. Our present study selected the collagenase-induced $\mathrm{ICH}$ rat model because collagenase-induced ICH in rat has a high mortality which is age-dependent with marked brain edema, like human ICH. This model has been suggested to better mimic human ICH syndrome [17, 18]. A comparison study [18] has shown that despite similar initial hematoma volumes, collagenaseinduced ICH resulted in a greater blood-brain barrier breakdown and more damage to the striatum, substantia nigra, white matter, and cortex. Tissue loss continued over 4 weeks in the collagenase model. In contrast, in the blood model, MRI revealed faster hematoma resolution, and little increase in the volume of tissue lost from 1 to 6 weeks. Moreover, functional deficits recovered more quickly and completely. Generally, the blood model mimics a single large bleed that was thought to occur in most ICH patients, but does not reproduce spontaneous bleeding. However, more recent clinical data suggest that bleeding continues for up to $24 \mathrm{~h}$ in many ICH patients. The rapid and apparently complete behavioral recovery in the blood model is at odds with the persistent and debilitating deficits seen in ICH patients. Furthermore, a rapid functional recovery is a significant problem in studies that seek to assess 
Fig. 4 Expression of selected miRNA in ICH rat model and human. a miR-124, miR-490, miR-873, and miR-708

expression patterns in the ICH rat brain and plasma. b miR-124, miR-490, miR-873, and miR-708 proportion in the human brain and hematopoietic organ relative to 147 human cells and tissues
A

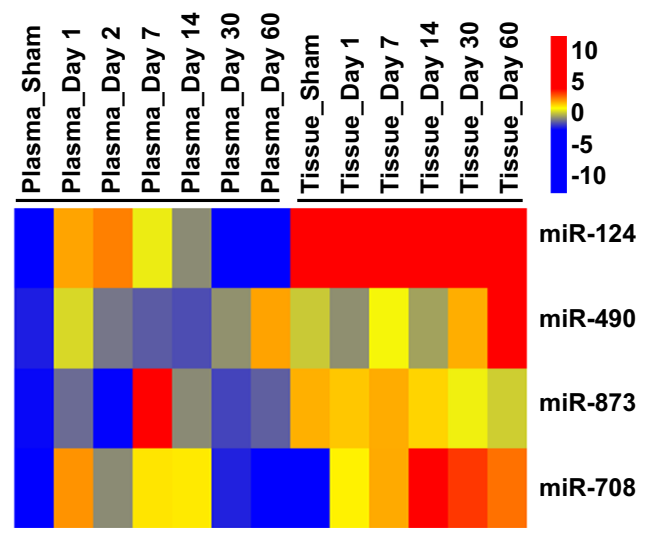

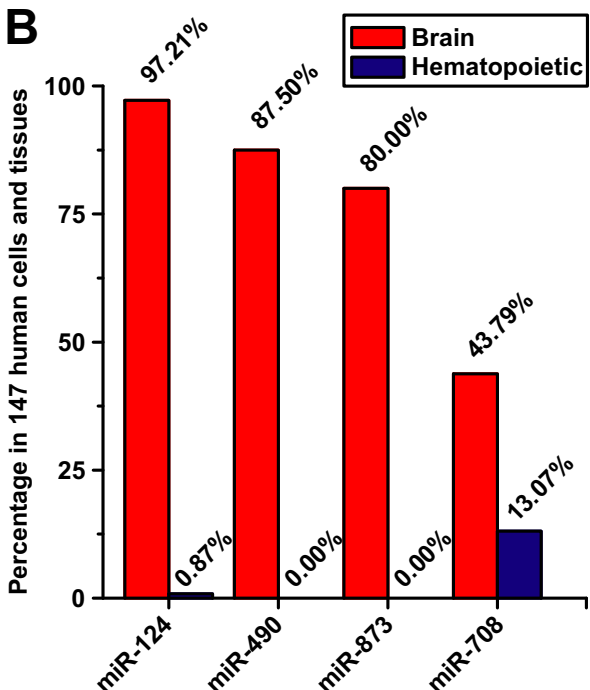

long-term outcome. Even though collagenase does not discriminate among different brain tissues, it produces massive bleeding and mortality, similar to that in human ICH. This model has been used extensively in investigations of $\mathrm{ICH}$ [23-30], including studies on the long-term deficits after collagenase-induced ICH [31].

Our results also suggest that plasma miRNAs are desirable biomarkers for $\mathrm{ICH}$. At present, the diagnosis and prognosis of stroke rely on imaging techniques such as magnetic resonance imaging. Biomarkers sampled from blood are particularly desirable [32]. An accurate and reliable blood biomarker for diagnosis and risk prediction of stroke is currently lacking. Several protein biomarkers have been suggested to be elevated on cerebral infarction. However, these markers are neither detected in the early phase of stroke nor are specific for brain injury. Ideal biomarkers should be easily accessible, reliable, cost-effective, and non-
Fig. 5 miR-124 expressed synchronously in plasma and brain tissue in the rat ICH model. a Relative plasma and tissue miR124 expression was measured by relative qPCR. Results are presented as group mean (SD) for the -fold change normalized to the miR-21 and miR-23b expression at a given time point. b Plasma miR-124 expression was measured by absolute qPCR. Left panel, standard curve generated by SYBR Green real-time PCR. Serially diluted standard commercial mature miR-124 was amplified. Threshold cycle $(\mathrm{Ct})$ values were plotted against copy number. Right panel, detection of miR-124 in human ICH patient plasma samples after the admission to the hospital

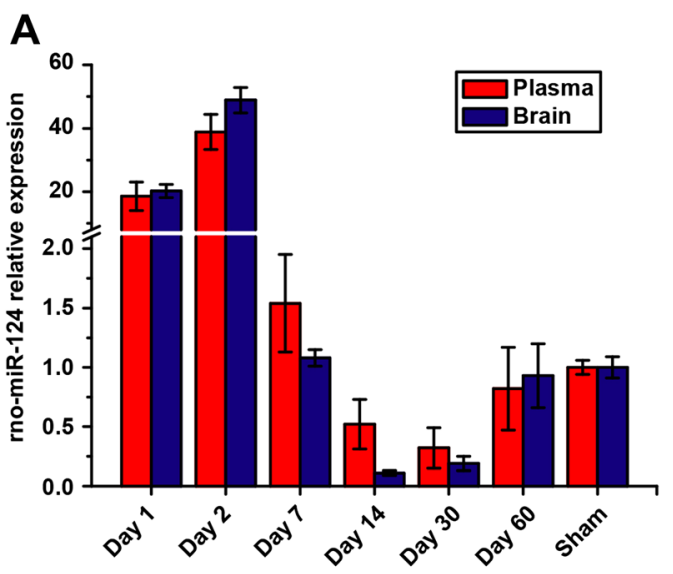

B
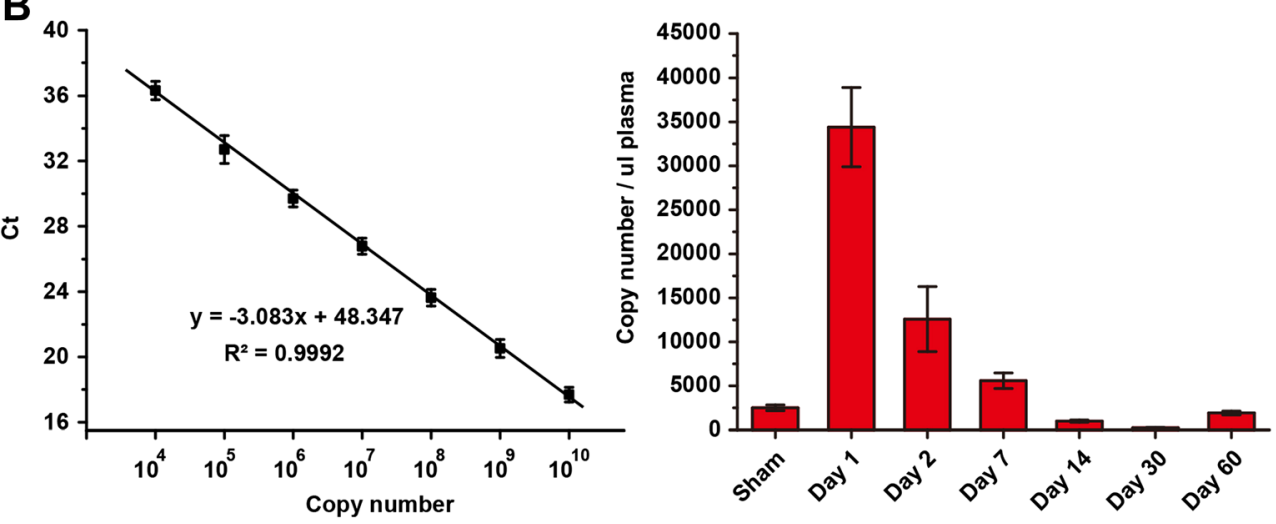
Table 2 Clinical information of health control and ICH patients

\begin{tabular}{lll}
\hline & $\begin{array}{l}\text { Health control } \\
(n=6)\end{array}$ & $\begin{array}{l}\text { ICH patients } \\
(n=20)\end{array}$ \\
\hline Mean age & $58.67 \pm 7.97$ & $59.75 \pm 9.53$ \\
Male & $3(50.0 \%)$ & $13(65.0 \%)$ \\
Hypertension & $1(16.7 \%)$ & $13(65.0 \%)$ \\
Smoker & $1(16.7 \%)$ & $12(60.0 \%)$ \\
Diabetics & $0(0.00 \%)$ & $5(25.0 \%)$ \\
Dyslipidemia & $1(16.7 \%)$ & $6(30.0 \%)$ \\
Previous stroke & $0(0.0 \%)$ & $2(10.0 \%)$ \\
\hline
\end{tabular}

invasive. As small and stable molecules, the plasma miRNAs are attractive biomarkers for human diseases. However, the expression pattern, function, and application of miRNAs in human ICH are not well understood.

The blood biomarkers were believed to be released into the circulation after injury [11]. However, the existence of bloodbrain barrier which strictly controls the exchanges between the blood and the brain makes the stroke biomarkers in blood difficult. The candidate macromolecule protein biomarkers of stroke were believed to depend on the disruption of the blood-brain barrier [33], which limit their application. Therefore, identification of biomarkers that could cross blood-brain barrier is highly desirable. Although the ability of 21 nt small molecule miRNA to cross the blood-brain barrier remains inconclusive, results from many published results including our present study suggest that they are likely able to cross the barrier.

In addition, it has been demonstrated that blood miRNAs remain stable upon exposure to severe conditions that induce almost immediate degradation of free RNA, such as boiling, very low or high $\mathrm{pH}$, and extended storage [34, 35]. The expression levels are reproducible and indicative of the disease state [36]. Blood miRNAs consistent among healthy individuals and can be detected in both plasma and serum [37].

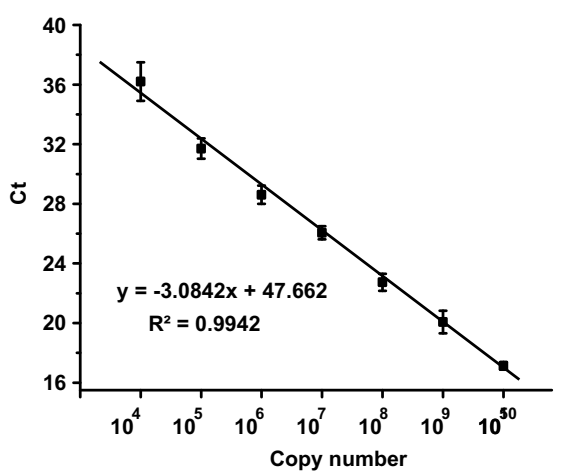

Fig. 6 miR-124 expressed in human ICH patient plasma samples after the admission to the hospital. Plasma miR-124 expression was measured by absolute qPCR. Left panel, standard curve generated by SYBR Green real-time PCR. Serially diluted standard commercial mature miR-124
It has been reported that plasma miRNAs are sensitive and specific biomarkers for various tissue injuries [11] and pathological conditions [11, 38-40].

Consistently, we used the collagenase-induced rat $\mathrm{ICH}$ model and evaluated the expression patterns of 728 rat miRNAs at different time points in rat brain tissues and plasma post-ICH. Results from this study revealed that a brain specific-enriched miRNA, miR-124, was expressed synchronously in brain tissue and plasma in the rat ICH stroke model. We further evaluated miR-124 expression pattern in the plasma samples of human ICH patient and showed that miR-124 exhibited similar expression pattern. Taken together, results from this study strongly suggest that changes of plasma concentration of miR-124 are promising biomarkers for early detection, diagnosis, and prognosis of human $\mathrm{ICH}$.

Previously, miR-124 has been suggested as a potential biomarker in tissue injury [11, 41] and cerebral infarction [31]. In this study, in the rat collagenase-induced ICH model and the plasma of human ICH patients, we showed that miR-124 is significantly up-regulated during acute injury phase, suggesting that it is derived from the injury of the brain. The plasma and brain expression levels were then decreased and even reduced on delayed recovery phase, suggesting the healing of the brain injury. Finally, it was returned to normal expression level when the rats were fully recovered.

The miR-124 is the most abundant miRNA in the brain. Due to its extraordinary enrichment in metazoan nervous systems through more than 500 Ma of evolution, miR-124 is one of the best-studied miRNAs in various organisms, by both basic biologists interested in molecular mechanisms of miRNA regulation and neuroscientists investigating brain development and function. As predicted by TargetScan and analyzed by DAVID, the target genes $(n=1808)$ of miR-124 mainly function in the regulation of transcription $(n=129)$ and nervous system development ( $n=57$ ) (Fig. 3b). These results suggest that miRNA-124 works through regulating the transcription of multiple pathways depending on the change of

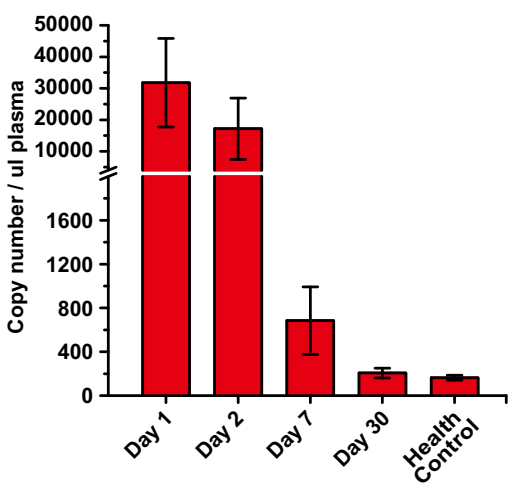

was amplified. Threshold cycle $(\mathrm{Ct})$ values were plotted against copy number. Right panel, detection of miR-124 in human ICH patient plasma samples after the admission to the hospital 
micro-environments. In addition, the top-ranking target gene is argonaute RISC catalytic component 2 (AGO2), which is related to cell stress. A highly significant correlation between AGO2 expression and cellular growth rate $(p<0.05)$ has been reported [42]. Therefore, it is possible that elevated miR-124 during early injury will markedly suppress AGO2 to inhibit cellular growth and direct cells to wound healing, while in the later recovery phase, reduced mi/r-124 could increase AGO2 to promote cell growth.

The possible clinical implications of plasma miR-124 pattern and concentrations in the detection, diagnosis, and prognosis of human ICH patients remain to be elucidated using larger number of patient samples. These include the possible correlation between brain edema size, the potential associations between the miRNA profiles and the gene expression profiles, as well as ICH grade and clinical outcome. In addition, the functions and mechanisms of miR-124 in ICH require further investigation to determine whether miR-124 could be used as a potential therapeutic target. Knowledge gained is especially important when investigating the nature of $\mathrm{ICH}$ pathogenesis. In addition, miRNAs involved in stroke may provide novel therapeutic targets for ICH.

Funding This study was funded by RGC (CUHK470911 to WSP and MCL), the National Natural Science Foundation of China (81773166 to Z.W.), the Basic Scientific Research Fund of Sun Yat-sen University (14ykpy41 to Z.W.), the Science and Technology Project of Guangdong (2017A020215098 to Z.W.; 2017A030313608 to Q.L.), and the Technology Planning Project of Guangzhou (No.201604020163 to Q.L.).

Compliance with Ethical Standards All procedures involving animals were performed to minimize pain or discomfort in accordance with current protocols approved by the Animal Research Ethics Committee of The Chinese University of Hong Kong.

Conflict of Interest The authors declare that they have no conflict of interest.

Open Access This article is distributed under the terms of the Creative Commons Attribution 4.0 International License (http:// creativecommons.org/licenses/by/4.0/), which permits unrestricted use, distribution, and reproduction in any medium, provided you give appropriate credit to the original author(s) and the source, provide a link to the Creative Commons license, and indicate if changes were made.

\section{References}

1. Amarenco P, Bogousslavsky J, Caplan LR, Donnan GA, Hennerici MG (2009) Classification of stroke subtypes. Cerebrovasc Dis 27(5):493-501. https://doi.org/10.1159/000210432

2. Burke TA, Venketasubramanian RN (2006) The epidemiology of stroke in the East Asian region: a literature-based review. Int $\mathrm{J}$ Stroke 1(4):208-215

3. Sudlow CL, Warlow CP (1997) Comparable studies of the incidence of stroke and its pathological types: results from an international collaboration. International Stroke Incidence Collaboration. Stroke 28(3):491-499
4. Qureshi AI, Mendelow AD, Hanley DF (2009) Intracerebral haemorrhage. Lancet 373(9675):1632-1644. https://doi.org/10.1016/ S0140-6736(09)60371-8

5. Chyatte D, Bruno G, Desai S, Todor R (1999) Inflammation and intracranial aneurysms. Neurosurgery 45(5):1137-1146

6. Bruno G, Todor R, Lewis I, Chyatte D (1998) Vascular extracellular matrix remodeling in cerebral aneurysms. J Neurosurg 89(3):431440. https://doi.org/10.3171/jns.1998.89.3.0431

7. Hara A, Yoshimi N, Mori H (1998) Evidence for apoptosis in human intracranial aneurysms. Neurol Res 20(2):127-130

8. Broderick JP (1993) Stroke trends in Rochester, Minnesota, during 1945 to 1984. Ann Epidemiol 3(5):476-479

9. Bartel DP (2009) MicroRNAs: target recognition and regulatory functions. Cell 136(2):215-233. https://doi.org/10.1016/j.cell. 2009.01.002

10. Xu J, Zhao J, Evan G, Xiao C, Cheng Y, Xiao J (2012) Circulating microRNAs: novel biomarkers for cardiovascular diseases. J Mol Med (Berl) 90(8):865-875. https://doi.org/10.1007/s00109-0110840-5

11. Laterza OF, Lim L, Garrett-Engele PW, Vlasakova K, Muniappa N, Tanaka WK, Johnson JM, Sina JF et al (2009) Plasma microRNAs as sensitive and specific biomarkers of tissue injury. Clin Chem 55(11):1977-1983. https://doi.org/10.1373/clinchem.2009.131737

12. Jeyaseelan K, Lim KY, Armugam A (2008) MicroRNA expression in the blood and brain of rats subjected to transient focal ischemia by middle cerebral artery occlusion. Stroke 39(3):959-966. https:// doi.org/10.1161/Strokeaha.107.500736

13. Dash PK, Redell JB, Liu Y (2009) Traumatic brain injury alters expression of hippocampal microRNAs: potential regulators of multiple pathophysiological processes. J Neurosci Res 87(6): 1435-1448. https://doi.org/10.1002/jnr.21945

14. Tang Y, Lu A, Aronow BJ, Wagner KR, Sharp FR (2002) Genomic responses of the brain to ischemic stroke, intracerebral haemorrhage, kainate seizures, hypoglycemia, and hypoxia. Eur J Neurosci 15(12):1937-1952

15. Zeng L, Liu J, Wang Y, Wang L, Weng S, Tang Y, Zheng C, Cheng Q et al (2011) MicroRNA-210 as a novel blood biomarker in acute cerebral ischemia. Front Biosci (Elite Ed) 3:1265-1272

16. Liu DZ, Tian Y, Ander BP, Xu H, Stamova BS, Zhan X, Turner RJ, Jickling $G$ et al (2010) Brain and blood microRNA expression profiling of ischemic stroke, intracerebral hemorrhage, and kainate seizures. J Cereb Blood Flow Metab 30(1):92-101. https://doi.org/ 10.1038/jcbfm.2009.186

17. Rosenberg GA, Munbryce S, Wesley M, Kornfeld M (1990) Collagenase-induced intracerebral hemorrhage in rats. Stroke 21(5):801-807

18. MacLellan CL, Silasi G, Poon CC, Edmundson CL, Buist R, Peeling J, Colbourne F (2008) Intracerebral hemorrhage models in rat: comparing collagenase to blood infusion. J Cereb Blood Flow Metab 28(3):516-525. https://doi.org/10.1038/sj.jcbfm. 9600548

19. Ng EK, Chong WW, Jin H, Lam EK, Shin VY, Yu J, Poon TC, Ng SS et al (2009) Differential expression of microRNAs in plasma of patients with colorectal cancer: a potential marker for colorectal cancer screening. Gut 58(10):1375-1381. https://doi.org/10.1136/ gut.2008.167817

20. Seo J, Shneiderman B (2002) Interactively exploring hierarchical clustering results. Computer 35(7):80-86

21. Landgraf P, Rusu M, Sheridan R, Sewer A, Iovino N, Aravin A, Pfeffer S, Rice A et al (2007) A mammalian microRNA expression atlas based on small RNA library sequencing. Cell 129(7):14011414. https://doi.org/10.1016/j.cell.2007.04.040

22. Rynkowski MA, Kim GH, Komotar RJ, Otten ML, Ducruet AF, Zacharia BE, Kellner CP, Hahn DK et al (2008) A mouse model of intracerebral hemorrhage using autologous blood infusion. Nat Protoc 3(1):122-128. https://doi.org/10.1038/nprot.2007.513 
23. Brown MS, Kornfeld M, Mun-Bryce S, Sibbitt RR, Rosenberg GA (1995) Comparison of magnetic resonance imaging and histology in collagenase-induced hemorrhage in the rat. J Neuroimaging 5(1):23-33

24. DeBow SB, Davies ML, Clarke HL, Colbourne F (2003) Constraint-induced movement therapy and rehabilitation exercises lessen motor deficits and volume of brain injury after striatal hemorrhagic stroke in rats. Stroke 34(4):1021-1026. https://doi.org/10. 1161/01.STR.0000063374.89732.9F

25. Del Bigio MR, Yan HJ, Buist R, Peeling J (1996) Experimental intracerebral hemorrhage in rats: magnetic resonance imaging and histopathological correlates. Stroke 27(12):2312-2319 discussion 2319-2320

26. Elger B, Seega J, Brendel R (1994) Magnetic resonance imaging study on the effect of levemopamil on the size of intracerebral hemorrhage in rats. Stroke 25(9):1836-1841

27. MacLellan C, Shuaib A, Colbourne F (2002) Failure of delayed and prolonged hypothermia to favorably affect hemorrhagic stroke in rats. Brain Res 958(1):192-200

28. Matsushita K, Meng W, Wang XY, Asahi M, Asahi K, Moskowitz MA, Lo EH (2000) Evidence for apoptosis after intracerebral hemorrhage in rat striatum. J Cereb Blood Flow Metab 20(2):396-404

29. Rosenberg GA, Navratil M (1997) Metalloproteinase inhibition blocks edema in intracerebral hemorrhage in the rat. Neurology 48(4):921-926

30. Rosenberg GA, Kornfeld M, Estrada E, Kelley RO, Liotta LA, Stetlerstevenson WG (1992) Timp-2 reduces proteolytic opening of blood-brain-barrier by type-IV collagenase. Brain Res 576(2):203-207

31. Chesney JA, Kondoh T, Conrad JA, Low WC (1995) Collagenaseinduced intrastriatal hemorrhage in rats results in long-term locomotor deficits. Stroke 26(2):312-316

32. Gilad S, Meiri E, Yogev Y, Benjamin S, Lebanony D, Yerushalmi N, Benjamin H, Kushnir M, Cholakh H, Melamed N, Bentwich Z, Hod M, Goren Y, Chajut A (2008) Serum microRNAs are promising novel biomarkers. PLoS One 3(9):e3148. doi: https://doi.org/ 10.1371/Journal.Pone.0003148

33. Kapural M, Krizanac-Bengez L, Barnett G, Perl J, Masaryk T, Apollo D, Rasmussen P, Mayberg MR et al (2002) Serum S-100 beta as a possible marker of blood-brain barrier disruption. Brain Res 940(1-2):102-104

34. Chen X, Ba Y, Ma LJ, Cai X, Yin Y, Wang KH, Guo JG, Zhang YJ et al (2008) Characterization of microRNAs in serum: a novel class of biomarkers for diagnosis of cancer and other diseases. Cell Res 18(10):997-1006. https://doi.org/10.1038/Cr.2008.282

35. Tsui NBY, Ng EKO, Lo YMD (2002) Stability of endogenous and added RNA in blood specimens, serum, and plasma. Clin Chem 48(10):1647-1653

36. Zhang CY, Chen X, Ba Y, Ma LJ, Cai X, Yin Y, Wang KH, Guo JG et al (2008) Characterization of microRNAs in serum: a novel class of biomarkers for diagnosis of cancer and other diseases. Cell Res 18(10):997-1006. https://doi.org/10.1038/cr.2008.282

37. Di Stefano V, Zaccagnini G, Capogrossi MC, Martelli F (2011) MicroRNAs as peripheral blood biomarkers of cardiovascular disease. Vasc Pharmacol 55(4):111-118. https://doi.org/10.1016/j.vph. 2011.08.001

38. Mitchell PS, Parkin RK, Kroh EM, Fritz BR, Wyman SK, Pogosova-Agadjanyan EL, Peterson A, Noteboom J et al (2008) Circulating microRNAs as stable blood-based markers for cancer detection. Proc Natl Acad Sci USA 105(30):10513-10518. https:// doi.org/10.1073/pnas.0804549105

39. Tijsen AJ, Creemers EE, Moerland PD, de Windt LJ, van der Wal AC, Kok WE, Pinto YM (2010) MiR423-5p as a circulating biomarker for heart failure. Circ Res 106(6):1035-U1063. https://doi. org/10.1161/Circresaha.110.218297

40. Wang K, Zhang SL, Marzolf B, Troisch P, Brightman A, Hu ZY, Hood LE, Galas DJ (2009) Circulating microRNAs, potential biomarkers for drug-induced liver injury. Proc Natl Acad Sci USA 106(11):4402-4407. https://doi.org/10.1073/pnas.0813371106

41. Weng HC, Shen CS, Hirokawa G, Ji X, Takahashi R, Shimada K, Kishimoto C, Iwai N (2011) Plasma miR-124 as a biomarker for cerebral infarction. Biomed Res-Tokyo 32(2):135-141

42. Lenkala D, Gamazon ER, LaCroix B, Im HK, Huang RS (2015) MicroRNA biogenesis and cellular proliferation. Transl Res 166(2): 145-151. https://doi.org/10.1016/j.trs1.2015.01.012 